\title{
Vaccine safety surveillance using routinely collected healthcare data - An empirical evaluation of epidemiological designs
}

Martijn J. Schuemie ${ }^{1,2,3,{ }^{*}}$, Faaizah Arshad ${ }^{1,3}$, Nicole Pratt ${ }^{4}$, Fredrik Nyberg ${ }^{5}$, Thamir M Alshammari ${ }^{6}$, George Hripcsak ${ }^{1,8}$, Patrick Ryan ${ }^{1,2,8}$, Daniel Prieto-Alhambra ${ }^{9,10}$, Lana YH Lai ${ }^{11}$, Xintong Li ${ }^{12}$, Stephen, Fortin $^{2}$, Evan Minty ${ }^{11}$, Marc A. Suchard ${ }^{1,3,13}$

Affiliations:

1. Observational Health Data Sciences and Informatics, New York, NY, USA

2. Observational Health Data Analytics, Janssen R\&D, Titusville, NJ, USA

3. Department of Biostatistics, University of California, Los Angeles, CA, USA

4. Quality Use of Medicines and Pharmacy Research Centre, Clinical and Health Sciences, University of South Australia, Australia

5. School of Public Health and Community Medicine, Institute of Medicine, Sahlgrenska Academy, University of Gothenburg, Gothenburg, Sweden

6. College of Pharmacy, Riyadh Elm University, Riyadh, Saudi Arabia

7. Real-World Evidence, Trial Form Support, Barcelona, Spain

8. Department of Biomedical Informatics, Columbia University, NY, NY, USA

9. Centre for Statistics in Medicine, NDORMS, University of Oxford, Oxford, UK

10. Department of Medical Informatics, Erasmus University Medical Center, Rotterdam, NL

11. O'Brien Institute for Public Health, Faculty of Medicine, University of Calgary, Canada

12. Division of Medical Sciences, University of Manchester, UK

13. Department of Human Genetics, University of California, Los Angeles, CA, USA

* Corresponding author:

Martijn Schuemie

schuemie@ohdsi.org

1125 Trenton Harbourton Road

Titusville, NJ 08560

United State of America 


\title{
Highlights
}

- Most methods used in vaccine safety surveillance show large type 1 error, which could lead to many false safety signals.

- Empirical calibration using effect-size estimates for negative control outcomes can restore type 1 error to close to nominal, often at the cost of marginal increases in type 2 error.

- After calibration, the self-controlled case series (SCCS) design shows the shortest time to detection for small true effect sizes, while the historical comparator appears best for large true effect sizes.

- Implementing negative control outcomes in a safety surveillance system is recommended to identify vulnerability to systematic error.

\section{Abstract}

Background: Routinely collected healthcare data such as administrative claims and electronic health records (EHR) can complement clinical trials and spontaneous reports when ensuring the safety of vaccines, but uncertainty remains about what epidemiological design to use.

Methods: Using 3 claims and 1 EHR database, we evaluate several variants of the case-control, comparative cohort, historical comparator, and self-controlled designs against historical vaccinations with real negative control outcomes (outcomes with no evidence to suggest that they could be caused by the vaccines) and simulated positive controls.

Results: Most methods show large type 1 error, often identifying false positive signals. The cohort method appears either positively or negatively biased, depending on the choice of comparator index date. Empirical calibration using effect-size estimates for negative control outcomes can restore type 1 error to close to nominal, often at the cost of increasing type 2 error. After calibration, the selfcontrolled case series (SCCS) design shows the shortest time to detection for small true effect sizes, while the historical comparator performs well for strong effects.

Conclusions: When applying any method for vaccine safety surveillance we recommend considering the potential for systematic error, especially due to confounding, which for many designs appears to be substantial. Adjusting for age and sex alone is likely not sufficient to address the differences between vaccinated and unvaccinated, and for the cohort method the choice of index date plays an important role in the comparability of the groups Inclusion of negative control outcomes allows both quantification of the systematic error and, if so desired, subsequent empirical calibration to restore type 1 error to its nominal value. In order to detect weaker signals, one may have to accept a higher type 1 error.

\section{Keywords}

vaccine safety, routinely collected data, adverse event, surveillance

\author{
Abbreviations \\ CCAE, IBM MarketScan Commercial Claims and Encounters \\ $\mathrm{Cl}$, Confidence Interval \\ COVID-19, COronaVIrus Disease 2019 \\ $\mathrm{CV}$, Critical Value \\ H1N1pdm, Hemagglutinin Type 1 and Neuraminidase Type 1 (2009 pandemic influenza) \\ HPV, Human papillomavirus \\ LLR, Log Likelihood Ratio \\ MaxSPRT, Maximum Sequential Probability Ratio Testing \\ MDCR, IBM MarketScan Medicare Supplemental Database
}


medRxiv preprint doi: https://doi.org/10.1101/2021.08.09.21261780; this version posted August 9, 2021. The copyright holder for this preprint (which was not certified by peer review) is the author/funder, who has granted medRxiv a license to display the preprint in perpetuity.

It is made available under a CC-BY 4.0 International license.

MDCD, IBM MarketScan Multi-State Medicaid Database

OHDSI, Observational Health Data Sciences and Informatics

PS, Propensity Score

SCCS, Self-Controlled Case Series

SCRI, Self-Controlled Risk Interval

TaR, Time-at-Risk 
medRxiv preprint doi: https://doi.org/10.1101/2021.08.09.21261780; this version posted August 9, 2021. The copyright holder for this preprint (which was not certified by peer review) is the author/funder, who has granted medRxiv a license to display the preprint in perpetuity.

It is made available under a CC-BY 4.0 International license .

\section{Introduction}

Vaccines are a critical part of the public health response to communicable disease. Given the extensive rate of vaccination among the general population, including otherwise healthy individuals, considerable emphasis is placed on ensuring vaccine safety. Post-marketing safety surveillance, therefore, is critical to ensure rare events not detectable in pre-market clinical trials due to limited sample size are detected early to maintain public confidence. Regulators frequently rely on spontaneous reports of safety concerns with vaccines; however, these data are often underreported[1] and lack accurate denominator information for calculation of population rates of disease.[2] It is, therefore, of critical importance that alternate data are used to implement rapid and rigorous identification of safety signals associated with vaccines.

Routinely collected healthcare data such as administrative claims and electronic health records (EHRs) may offer information that is complementary to clinical trials and spontaneous reports. Although these data were not collected for the purpose of safety surveillance, several epidemiological designs for analyzing longitudinal healthcare data to this end have been proposed. Some of these methods were proposed as 'signal generation' methods, while others have been considered for 'signal confirmation',[3] where the main distinction seems to be the complexity of the method and its ability to scale from a single vaccine-outcome pair to many. However, recent developments in large-scale analytics such as large-scale propensity scores (PS),[4] and open-source analytics software like that developed by the Observational Health Data Sciences and Informatics (OHDSI),[5] mean the distinction between signal generation and confirmation has become blurred. It is, for example, perfectly feasible to use propensity score adjustment on a large scale, and, therefore, to use it efficiently for signal generation.

Given the current coronavirus disease 2019 (COVID-19) pandemic and mass vaccination, there is great interest in how best to monitor the safety of these vaccines. Prior research has shown that the four most commonly used epidemiological designs in vaccine safety surveillance were cohort studies, casecontrol studies, self-controlled case series (SCCS), and self-controlled risk-intervals (SCRI). [3, 6] One simulation study comparing the four designs indicated cohort study designs had the best performance in the sequential analysis of vaccine safety surveillance, with the lowest false positive rate, highest empirical power, and smallest risk estimate bias.[7] The SCCS and SCRI study designs were also efficient alternatives. This study, however, did not account for the potential for misclassification or confounders, such as age or seasonal effects. Another simulation study that used the cohort design as a benchmark concluded that the estimates of the case-control, SCCS and SCRI were within 5\% of the true risk parameters.[8] Of the four study designs, the case-control estimates were biased by fixed confounding and less precise. While the estimates of the SCCS may be biased by unadjusted seasonal confounding, it was found to be an efficient alternative to the cohort study design, with the additional ability to avoid unmeasured between-person confounding by its self-controlled nature.

There is, however, a research gap on whether the findings from these simulation studies would apply to real-world data. To address this, we used data from three insurance claims databases and one EHR database from the United States to evaluate and compare a selection of safety surveillance methods. We studied the association between retrospective vaccinations and real outcomes assumed not to be causally related to vaccines (negative control outcomes), as well as imputed positive controls (outcomes simulated to be caused by the vaccines) to evaluate method performance. 
medRxiv preprint doi: https://doi.org/10.1101/2021.08.09.21261780; this version posted August 9, 2021. The copyright holder for this preprint (which was not certified by peer review) is the author/funder, who has granted medRxiv a license to display the preprint in perpetuity.

It is made available under a CC-BY 4.0 International license .

\section{Material and methods}

\subsection{Vaccines of interest}

Our evaluation focuses on six existing (groups of) vaccines, representing different scenarios, such as a response to a pandemic, seasonal vaccinations, and continuous vaccination programs. We follow each vaccine for specific time periods (start date to end date), as shown in Table 1. For seasonal flu, we included analyses for all vaccines used during that season combined, as well as separate analyses for specific flu. For some methods, a period prior to the vaccine study period (historic start to historic end date) is used to estimate the historic incidence rate. Codes to define each vaccine group can be found in the Supplementary Materials.

Table 1

Exposures of interest.

\begin{tabular}{lllll}
$\begin{array}{l}\text { Exposure Name } \\
\text { H1N1pdm vaccination }\end{array}$ & Start Date & End Date & $\begin{array}{l}\text { History Start } \\
\text { Date }\end{array}$ & $\begin{array}{l}\text { History End } \\
\text { Date }\end{array}$ \\
\hline Seasonal flu vaccination (Fluvirin) & $01-09-2009$ & $31-05-2010$ & $01-09-2008$ & $31-05-2009$ \\
\hline Seasonal flu vaccination (Fluzone) & $01-09-2017$ & $31-05-2018$ & $01-09-2016$ & $31-05-2017$ \\
\hline Seasonal flu vaccination (All) & $01-09-2017$ & $31-05-2018$ & $01-09-2016$ & $31-05-2017$ \\
\hline Zoster vaccination (Shingrix) & $01-01-2018$ & $31-12-2018$ & $01-09-2016$ & $31-05-2017$ \\
\hline HPV vaccination (Gardasil 9) & $01-01-2018$ & $31-12-2018$ & $01-01-2017$ & $31-12-2017$ \\
\hline
\end{tabular}

The varicella-zoster and Human papillomavirus (HPV) vaccines require two doses. In this paper, we do not stratify by dose; anyone receiving a second dose is included in the analysis twice, with separate index dates for the two vaccinations.

\subsection{Negative control outcomes}

Negative controls are outcomes for which there is no evidence to suggest that they could be causally related to any of the vaccines, and, therefore, ideally would not be flagged as a signal by a safety surveillance system. A single set of negative control outcomes was defined for all six vaccine groups. To identify negative control outcomes that match the severity and prevalence of suspected vaccine adverse effects, a candidate list of negative controls was generated based on similarity of prevalence and percent of diagnoses that were recorded in an inpatient setting (as a proxy for severity). Manual review of this list by clinical experts created the final list of 93 negative control outcomes (see Supplementary Materials). Negative control outcomes are defined as the first occurrence of the negative control concept or any of its descendants.

\subsection{Imputed positive control outcomes}

Positive controls are outcomes known to be caused by vaccines, and ideally would be detected as signals by a safety surveillance system as early as possible. However, real positive controls are problematic for various reasons.[9] First, vaccine adverse effects that are well established are rare. Second, even when an effect is established, the magnitude is never known with precision. Third, for well-established adverse effects, actions are often taken to mitigate the risk, such as careful monitoring or even restricting use of the vaccine, masking these effects in real-world data. In our study, we therefore do not 
medRxiv preprint doi: https://doi.org/10.1101/2021.08.09.21261780; this version posted August 9, 2021. The copyright holder for this preprint (which was not certified by peer review) is the author/funder, who has granted medRxiv a license to display the preprint in perpetuity.

It is made available under a CC-BY 4.0 International license .

use real positive controls. To still assess the type 2 error one could expect for a given method, we instead use a simple simulation approach: for every negative control effect-size estimate produced by a method in a database, we impute three positive controls by multiplying the estimated effect size by 1.5 , 2 , and 4 respectively. For example, if for a negative control outcome (having true effect size $=1$ ) a casecontrol design produces an odds ratio of 1.1, we can impute a positive control estimate (having true effect size $=1.5$ ) for that design as $1.1 * 1.5=1.65$. This simulation approach makes strong assumptions about the nature of the systematic error, most importantly that systematic error does not change as a function of true effect size.

\subsection{Data sources}

This study uses data from each of the following four observational healthcare databases:

The IBM MarketScan Commercial Claims and Encounters (CCAE) database contains adjudicated health insurance claims (e.g., inpatient, outpatient, and outpatient pharmacy) from large employers and health plans who provide private healthcare coverage to employees, their spouses and dependents.

The IBM MarketScan Medicare Supplemental Database (MDCR) database contains adjudicated health insurance claims of retirees with primary or Medicare supplemental coverage through privately insured fee-for-service, point-of-service or capitated health plans.

The IBM MarketScan Multi-State Medicaid Database (MDCD) database contains adjudicated health insurance claims for Medicaid enrollees from multiple states and includes hospital discharge diagnoses, outpatient diagnoses and procedures, and outpatient pharmacy claims.

The Optum ${ }^{\circledR}$ de-identified Electronic Health Record dataset (Optum EHR) contains clinical information, prescriptions, lab results, vital signs, body measurements, diagnoses and procedures derived from clinical notes from both inpatient and outpatient environments using natural language processing.

All data were converted to the Observational Medical Outcomes Partnership (OMOP) Common Data Model v5.3.1.

\subsection{Evaluated method variations}

The following method variations were evaluated, each using a time-at-risk (TaR) window of 1-28 days relative to the date of vaccination (both first and second dose, where applicable). For more details, see the Supplementary Materials.

- Case-control: The case-control design compares cases (those with the outcome) to controls (those that do not have the outcome on or before the index date), and looks back in time for exposures to a vaccine. We select up to four controls per case. We evaluate two variations:

- Age \& sex matched controls, with the index date of controls set to the date of the outcome of the case to which they are matched.

- Age \& sex adjusted, using random controls, with the index dates of the controls sampled from the distribution of outcome dates of the cases.

- (Concurrent) Cohort method: A comparative cohort study most closely emulates a randomized clinical trial, comparing the target cohort (those vaccinated) to some comparator (nonvaccinated) cohort. We define two types of comparator cohorts: one having an outpatient visit on the index date and another having a random date as the index date. For unadjusted analyses we sample a comparator cohort of equal size to the vaccinated cohort, for adjusted comparisons 
we sample cohorts of four times the size of the vaccinated cohort (two times for H1N1pdm) to account for loss of power due to adjustment. We exclude subjects from the comparator cohort who had a vaccination for the same disease as the target vaccine within the vaccine study period, on or before the index date. Propensity models use a large generic set of covariates, including demographics and covariates per drug, condition, procedure, measurement, etc., and are fitted using large-scale regularized regression as described previously. [10] Per-month PS matching uses only the vaccinated during that month and their comparators to create a PS and perform matching, and this matching is preserved in subsequent months. We evaluate 10 variations:

- Unadjusted, outpatient visits as comparator index

- PS matching, outpatient visits as comparator index

- Unadjusted, random days as comparator index

- PS matching, random days as comparator index

- PS stratification, outpatient visits as comparator index

- PS stratification, random days as comparator index

- PS weighting, outpatient visits as comparator index

- PS weighting, random days as comparator index

- Per-month PS matching, outpatient visits as comparator index

- Per-month PS matching, random days as comparator index

The per-month PS matching variations were only executed for the H1N1 vaccines for computational reasons.

- Historical comparator cohort design: Traditionally, vaccine surveillance methods compute an expected count based on the incidence rate estimated during some historic time period, for example in the years prior to the initiation of the surveillance study. We use the historic period indicated in Table 1. Because this method proved sensitive to changes in coding practice over time, we defined variants marked as 'filtered' below which we filtered outcomes where the change in overall incidence rate was greater than $50 \%$ when comparing the historic period to the surveillance period so far. In total, we evaluate eight variations:

○ Unadjusted, using the entire historic period

- Age \& sex adjusted, using the entire historic period

- Unadjusted, using the TaR after a random outpatient visit during the historic period

- Age \& sex adjusted, using the TaR after a random outpatient visit during the historic period

- Unadjusted, using the entire historic period, filtered

- Age \& sex adjusted, using the entire historic period, filtered

- Unadjusted, using the TaR after a random outpatient visit during the historic period, filtered

- Age \& sex adjusted, using the TaR after a random outpatient visit during the historic period, filtered

- Self-Controlled Case Series (SCCS) / Self-Controlled Risk Interval (SCRI): The SCCS and SCRI designs are self-controlled, comparing the TaR (the time shortly following the vaccination) to some other time in the same patient's record. The SCCS design uses all available patient time in the vaccine study period when not at risk as the control time. [10] To account for the fact that people are either more or less likely to be vaccinated directly after a serious health outcome, the 30 days prior to vaccination are removed from the analysis. Adjustment for age and season uses 5-knot bicubic splines. The SCRI design uses a pre-specified control interval relative to the 
medRxiv preprint doi: https://doi.org/10.1101/2021.08.09.21261780; this version posted August 9, 2021. The copyright holder for this preprint (which was not certified by peer review) is the author/funder, who has granted medRxiv a license to display the preprint in perpetuity.

It is made available under a CC-BY 4.0 International license .

vaccination date as the control time. [9] This unexposed time can be either before or after the

TaR. We evaluate five variations:

- Unadjusted SCCS excluding a 30-day pre-vaccination window

- Age \& season adjusted SCCS excluding a pre-vaccination window

- SCRI with a control interval of 43 to 15 days prior to vaccination

- SCRI with a control interval of 43 to 71 days after to vaccination

- Unadjusted SCCS excluding all pre-vaccination time, so including all time after day 28 (the end of the TaR) as control time.

To adjust for multiple testing when testing the same hypothesis sequentially we apply maximum sequential probability ratio testing (MaxSPRT) to all methods by computing the log likelihood ratio (LLR) as well as a critical value for the observed power and alpha of 0.05.[11] Critical values were computed using the 'Sequential' R package version 3.3.1, using the Poisson model for the historical comparator design, and the binomial model for all other designs.[12]

To adjust for systematic error, we apply an empirical calibration procedure described elsewhere $[13,14]$ that attempts to restore $p$-values and LLRs to nominal (e.g. ensuring that after calibration approximately $5 \%$ of negative controls have $p<0.05$ ). In short, this procedure first estimates the distribution of systematic error, assumed to be Gaussian, using the observed estimates for negative controls. Using the estimated distribution, we then generate calibrated $p$-values and LLRs considering both random and systematic error. Typically, but not necessarily, the calibrated $p$ is higher than the nominal $p$, reflecting the problems unaccounted for in the standard procedure (such as unmeasured confounding, selection bias, and measurement error) but accounted for in the calibration. For the purpose of this evaluation we apply a leave-one-out approach, calibrating the estimate for a control outcome using the systematic error distribution fitted on all control outcomes except the one being calibrated.

\subsection{Performance metrics}

To evaluate timeliness, time was divided into one-month periods, where for each month the data collected in that month and those preceding it were used to compute estimates. For each database vaccine group - outcome - method - period combination we compute the uncalibrated and calibrated effect-size estimate (odds ratio, hazard ratio or incidence ratio) with 95\% confidence interval (Cl) and one-sided p-value, as well the LLR and MaxSPRT critical value.

Based on these statistics, for each database - vaccine group - method, we derive the systematic error distribution for negative controls, [14] type 1 error (how often the null is rejected when the null is true), type 2 error (how often the null is not rejected when the null is not true), and time (months) to $50 \%$ sensitivity (i.e., $50 \%$ of positive controls flagged as statistically significant) stratified by true effect size. Note that we changed this metric from the initially intended $80 \%$ sensitivity, which corresponds to a more common target, to $50 \%$ sensitivity, because almost no method and database achieved $80 \%$ sensitivity in the study period. Plots showing time to $80 \%$ sensitivity are included in the Supplementary Materials.

\subsection{Open science}

The protocol as well as the analytic source code used to execute this study are available at https://github.com/ohdsi-studies/Eumaeus. The protocol has also been registered at ENCEPP with registration number EUPAS40259. 
medRxiv preprint doi: https://doi.org/10.1101/2021.08.09.21261780; this version posted August 9, 2021. The copyright holder for this preprint (which was not certified by peer review) is the author/funder, who has granted medRxiv a license to display the preprint in perpetuity.

It is made available under a CC-BY 4.0 International license.

\section{Results}

Where feasible, we executed all 25 method variations on all six vaccine groups, negative and positive controls, and time periods against the four databases, briefly characterized in Table 2, thus producing a total of 1,380,672 effect size estimates. From these we derive a large set of performance metrics, which vary depending on choices of which control outcomes and data to include in the evaluation. Below we present several examples of our results, starting with a single control, single database and two analysis variants, and gradually increasing the complexity. However, it is infeasible to cover the full set of results, and instead we refer the reader to the Supplementary Materials.

\section{Table 2}

Database characteristics and vaccination counts during the vaccination study period.

\begin{tabular}{|lrrrr|}
\hline Characteristic & \multicolumn{1}{c}{ CCAE } & MDCD & MDCR & Optum EHR \\
\hline Total number of subjects & $156,628,301$ & $31,355,646$ & $10,180,158$ & $97,936,862$ \\
\hline Fraction female & $51.10 \%$ & $56.20 \%$ & $55.30 \%$ & $53.60 \%$ \\
\hline Fraction male & $48.90 \%$ & $43.80 \%$ & $44.70 \%$ & $46.40 \%$ \\
\hline H1N1pdm vaccinations & 753,592 & 206,865 & 12,913 & 156,974 \\
\hline Seasonal flu vaccinations (Fluvirin) & 119,242 & 15,288 & 822 & 14,829 \\
\hline Seasonal flu vaccinations (Fluzone) & 957 & 3,358 & 34,414 & 355,593 \\
\hline Seasonal flu vaccinations (All) & $3,517,021$ & $1,237,934$ & 264,636 & $2,617,230$ \\
\hline 1st HPV vaccinations (Gardasil 9) & 376,795 & 237,008 & 8 & 244,664 \\
\hline 2nd HPV vaccinations (Gardasil 9) & 49,543 & 15,156 & 0 & 29,579 \\
\hline 1st Zoster vaccinations (Shingrix) & 148,541 & 11,431 & 52,877 & 221,938 \\
\hline 2nd Zoster vaccinations (Shingrix) & 72,518 & 5,405 & 30,364 & 64,187 \\
\hline
\end{tabular}

\subsection{An example control outcome for one vaccine}

We illustrate our experiment with a single negative control outcome: H1N1pdm vaccines and contusion of toe. We study this relationship in the Optum EHR database, using all 9 months of data in our study period (September 2009 to May 2010), during which we observe 156,467 vaccinations. Our TaR is 1-28 days relative to the date of vaccination.

We can estimate the effect size using an unadjusted historical comparator design, using the entire historic period (September 2008 to May 2009) to estimate the background rate. During this historic period, the observed incidence rate (IR) in the Optum EHR database is 0.29 per 1,000 patient years. Based on the number of H1N1pdm vaccinations and TaR, this generates an expected count of 3.4. We observe 14 cases during TaR, leading to an incidence rate ratio (IRR) of 4.08 (95\% Cl: $2.30-6.62$ ) and a LLR of 9.12, which exceeds the critical value of 1.73 computed for this analysis.

Alternatively, we could estimate the effect size using the SCCS design, adjusting for age and season, and excluding a pre-vaccination window from analysis. The total number of cases observed during the study period is 2,770 . For cases that were vaccinated, 116 experienced the outcome outside the TaR, and 14 experienced the outcome during the TaR, leading to an IRR of 1.07 (95\% Cl: $0.59-1.81)$ and LLR of 0.03, which does not exceed the critical value of 1.73 . 
medRxiv preprint doi: https://doi.org/10.1101/2021.08.09.21261780; this version posted August 9, 2021. The copyright holder for this preprint (which was not certified by peer review) is the author/funder, who has granted medRxiv a license to display the preprint in perpetuity.

It is made available under a CC-BY 4.0 International license .

These results demonstrate that using different methods to answer the same question with the same data can lead to heterogeneous estimates, and potentially to different regulatory decisions. This is further illustrated in Fig. 1, showing the effect size estimates of all method variations for our example negative control outcome (i.e. true effect size is assumed to be 1). Note that some methods were unable to produce an estimate, for various reasons. For example, the unadjusted cohort method design using a random-day comparator found no occurrences of the outcome during the TaR in the comparator.

Analysis choices
Case-control
Age \& sex matched controls
Age \& sex adjusted, using random controls
Cohort method
Unadjusted, using outpatient visits as comparator
PS matching, using outpatient visits as comparator
Unadjusted, using random days as comparator
PS matching, using random days as comparator
PS stratification, using outpatient visits as comparator
PS stratification, using random days as comparator
PS weighting, using outpatient visits as comparator
PS weighting, using random days as comparator
Per-month PS matching, using outpatient visits as comparator
Per-month PS matching, using random days as comparator
Historical comparator
Unadjusted, using entire historic period
Age \& sex adjusted, using entire historic period
Unadjusted, using TaR after historic visit
Age \& sex adjusted, using TaR after historic visit
Unadjusted, using entire historic period, filtered
Age \& sex adjusted, using entire historic period, filtered
Unadjusted, using TaR after historic visit, filtered
Age \& sex adjusted, using TaR after historic visit, filtered
SCCS / SCRI
Unadjusted SCCS excluding pre-vaccination window
Age \& season adjusted SCCS excluding pre-vaccination window
SCRI with prior control interval
SCRI with posterior control interval
Unadjusted SCCS excluding all pre-vaccination time

Effect size $(95 \% \mathrm{Cl}) \quad$ LLR

$3.33(0.64-15.44) \quad 1.09$

$2.40(0.49-9.81) \quad 0.65$

$3.48(1.25-12.29) \quad 2.92^{*}$

$1.50(0.54-4.47) \quad 0.30$

$-$

$2.00(0.81-4.86)$

$-\quad-$

$4.42(1.83-10.42) \quad 5.09^{\star}$

$0.97(0.18-5.26)$

-

$4.08(2.30-6.62)$

$4.18(2.35-6.79)$

$3.35(1.85-5.58)$

$3.50(1.92-5.87)$

$4.08(2.30-6.62)$

$4.18(2.35-6.79)$

$3.35(1.85-5.58)$

$3.50(1.92-5.87)$

$1.01(0.56-1.71)$

$1.07(0.59-1.81)$

$1.21(0.56-2.66)$

$1.31(0.60-2.96)$

$0.95(0.51-1.64)$

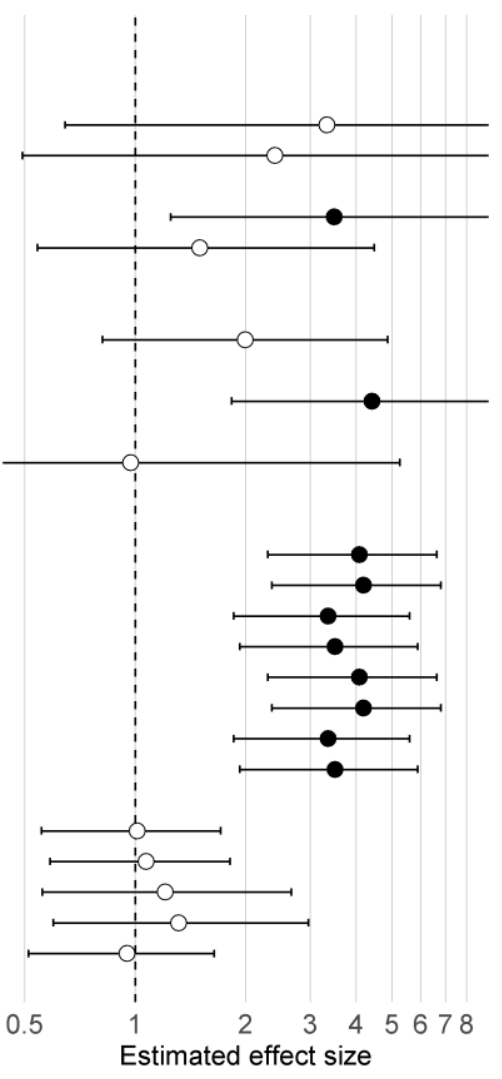

Fig. 1. Effect size estimates, $95 \% \mathrm{Cl}$, and LLRs for one example control. We use each analysis variation to estimate the causal effect size of H1N1pdm vaccination on the risk of 'contusion of toe' in the Optum EHR database, using the data across all 9 months. The true effect size is 1 , as indicated by the dashed line. ' ${ }^{* \prime}$ and filled dots indicates the LLR exceeds the critical value. $\mathrm{Cl}=$ Confidence Interval, $\mathrm{LLR}=\mathrm{Log}$ Likelihood Ratio, TaR = Time-at-Risk.

\subsection{Extending the example to all negative control outcomes for one vaccine}

This process was repeated for all negative control outcomes. The top row in Fig. 2 shows a compact representation of the effect size estimates of four example method variations for the negative controls, where the true effect size is assumed to be 1.

Fig. 2 shows that some methods tend to overestimate the effect size, rejecting the null when the null is assumed true more often than expected by chance alone ( $5 \%$ at an alpha of 0.05 ). Because we have a large collection of negative controls, we can use these to fit a systematic error distribution as shown in the bottom row of Fig. 2. One way to think of this distribution is that it is the distribution needed to explain the difference between the observed spread of the negative control estimates and expected spread based on random error alone. If the spread can be completely explained by random error as 
quantified by each method's standard error, the systematic error distribution will have a mean and standard deviation of 0 . Another way to think of this distribution is that for a future study using the same method and data, but a new outcome, the systematic error in that study will draw from this systematic error distribution.
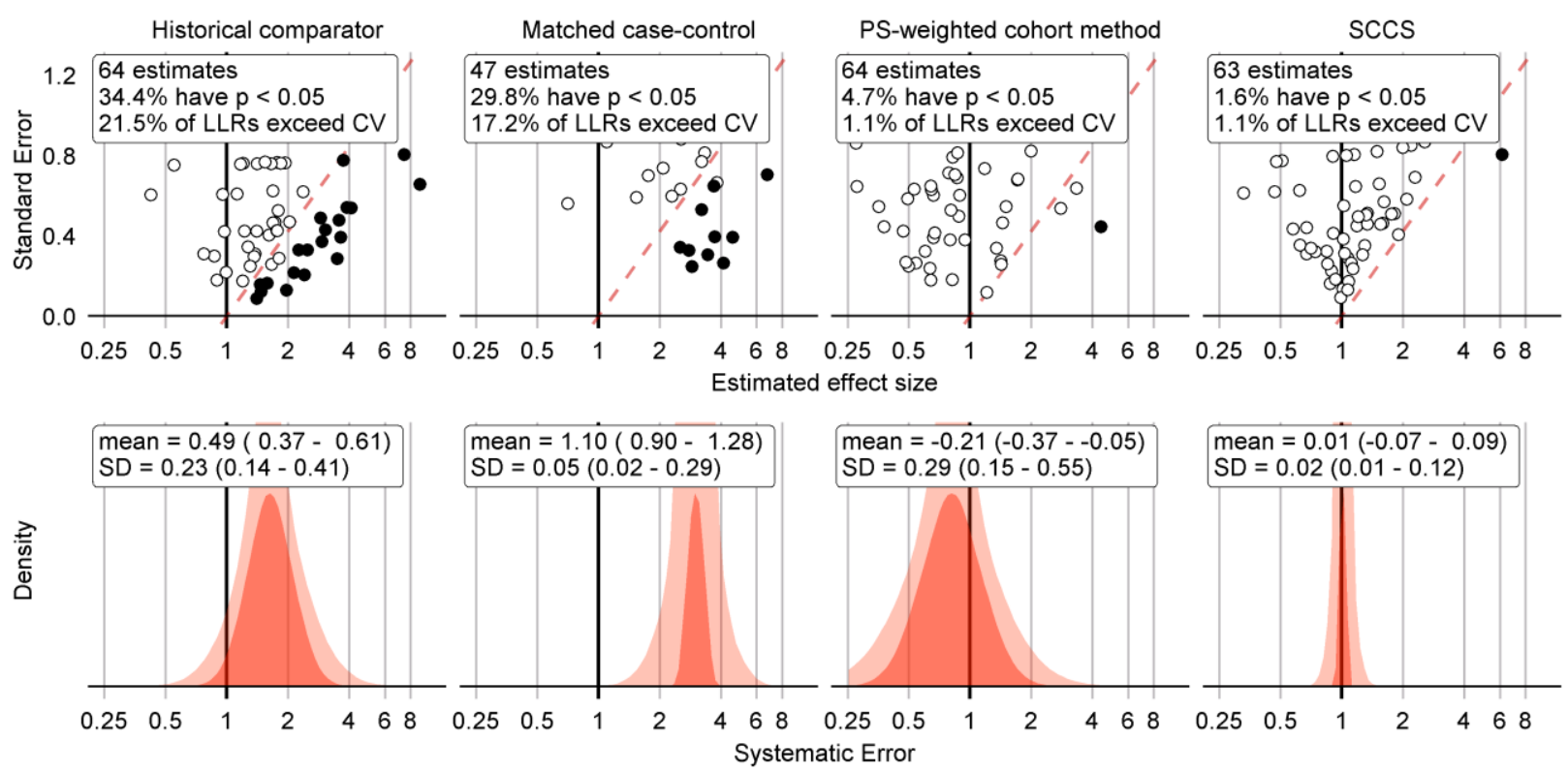

Fig. 2. Negative control outcome effect size estimates and fitted systematic error distributions for four example method variations. In the top row, dots indicate the estimated effect size (x-axis) and corresponding standard error ( $y$-axis), which is linearly related to the width of the confidence interval. Estimates below the red dashed line have a one-sided $p$-value $<0.05$, and filled dots indicate the LLR exceeds the CV. The bottom row shows the systematic error distributions fitted using the negative control estimates above, for the maximum likelihood estimates of the parameters (red area), and the 95\% credible interval (pink area). The historical comparator variant adjusts for age and sex, and uses the TaR after a historic outpatient visits to estimate the background rate. The case-control design matches up to 4 controls per case on age and sex. The cohort method design uses PS weighting and outpatient visits as comparator index date. The SCCS design adjusts for age and season and excludes a prevaccination window of 30 days from analysis. CV = Critical Value, LLR $=$ Log Likelihood Ratio, SCCS = SelfControlled Case Series, SD = Standard Deviation, PS = Propensity Score.

\subsection{Systematic error based on control outcomes across vaccines and methods}

Applying this procedure to all method variations and vaccines in the Optum EHR database produces Fig. 3. For the other databases please see the Supplementary Materials. As illustrated by the plot, casecontrol methods and historical comparator analyses tend to be positively biased, with many negative control outcomes identified as potential safety signals in most scenarios before calibration. The cohort method appears either positively or negatively biased, depending on the choice of comparator index date. SCCS/SCRI seem less biased, with systematic error more evenly and more narrowly distributed around the null. 

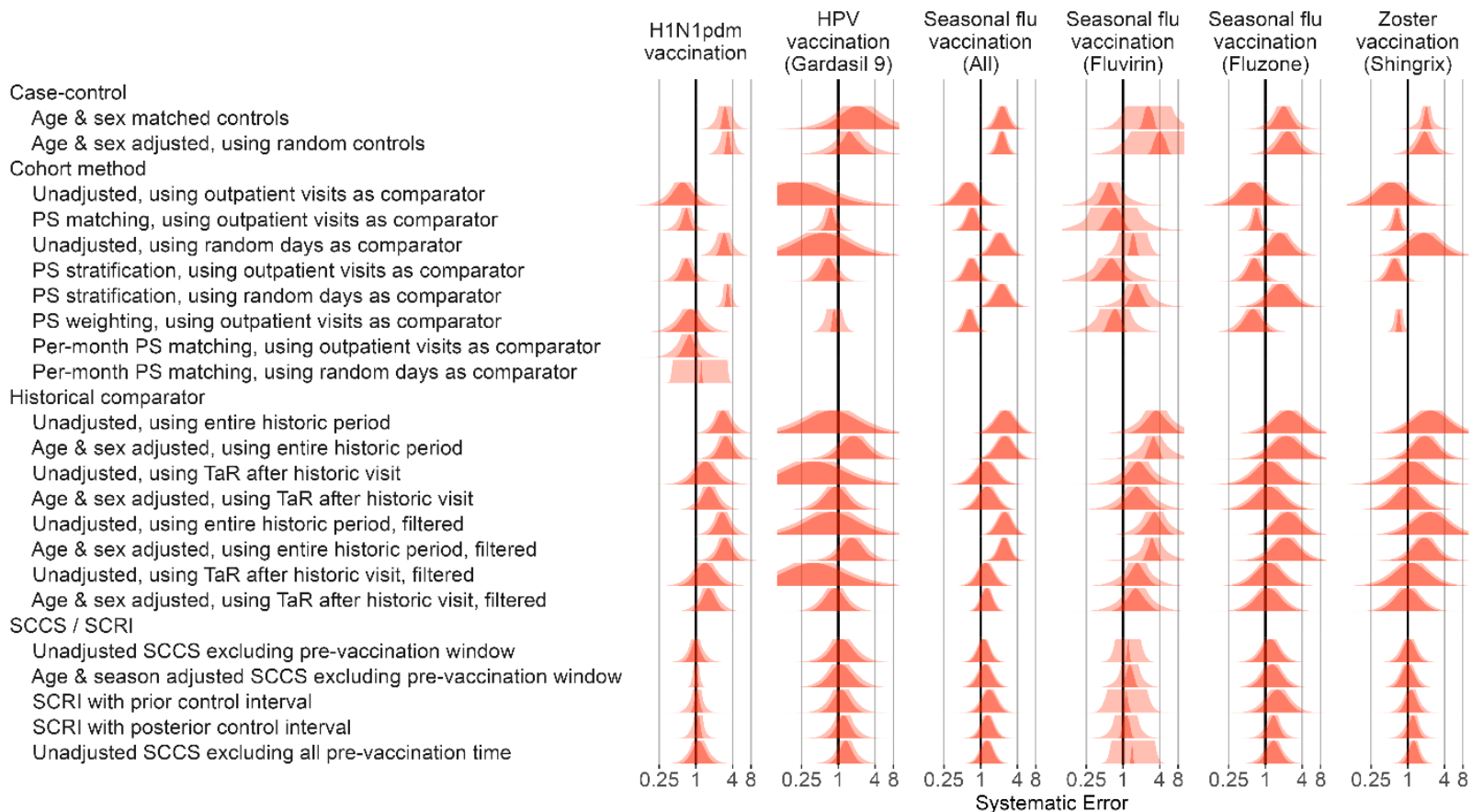

Fig. 3. Fitted systematic error distributions. For each method variation and vaccine group, the systematic error distribution fitted on the negative control estimates in the Optum EHR database are shown. The red area indicates the maximum likelihood estimates of the distribution parameters. The pink area indicates the $95 \%$ credible interval. HPV = Human papillomavirus, PS = Propensity Score, SCCS $=$ Self-Controlled Case Series, SCRI = Self-Controlled Risk Interval, TaR = Time-at-Risk.

\subsection{Type 1 and 2 error tradeoffs}

In addition to the systematic error inherent to a method applied to a database for a particular exposure, we consider the random error, or in other words the statistical power of a method. The estimates computed for our imputed positive control outcomes allow us to evaluate how often the null is rejected when the null is false, and thus compute type 2 error. Both type 1 and 2 error are shown in Fig. 4.

Comparing type 1 and 2 error of different method variations can be complicated when both vary. Moreover, type 1 and 2 error are interchangeable, for example by changing the alpha threshold, presenting a moving target. To facilitate the comparison of methods, we apply empirical calibration, a process that uses the fitted systematic error distribution to restore the type 1 error to its nominal value (of $5 \%$ at an alpha of 0.05 ). [14] This typically increases type 2 error, depending on how much systematic error needs to be adjusted for in the calibration. Fig. 4 shows the type 1 and 2 error both before and after empirical calibration of the $\mathrm{p}$-value. Overall, SCCS/SCRI and cohort methods provide the best combination of type $1+$ type 2 error, whilst historical comparison and case-control lead to much higher type 1 error in most scenarios. 
medRxiv preprint doi: https://doi.org/10.1101/2021.08.09.21261780; this version posted August 9, 2021. The copyright holder for this preprint (which was not certified by peer review) is the author/funder, who has granted medRxiv a license to display the preprint in perpetuity.

It is made available under a CC-BY 4.0 International license .

Case-control

Age \& sex matched controls

Age \& sex adjusted, using random controls

Cohort method

Unadjusted, using outpatient visits as comparator

PS matching, using outpatient visits as comparator

Unadjusted, using random days as comparator

PS matching, using random days as comparator

PS stratification, using outpatient visits as comparator

PS stratification, using random days as comparator

PS weighting, using outpatient visits as comparator

PS weighting, using random days as comparator

Per-month PS matching, using outpatient visits as comparator

Per-month PS matching, using random days as comparator

Historical comparator

Unadjusted, using entire historic period

Age \& sex adjusted, using entire historic period

Unadjusted, using TaR after historic visit

Age \& sex adjusted, using TaR after historic visit

Unadjusted, using entire historic period, filtered

Age \& sex adjusted, using entire historic period, filtered

Unadjusted, using TaR after historic visit, filtered

Age \& sex adjusted, using TaR after historic visit, filtered SCCS / SCRI

Unadjusted SCCS excluding pre-vaccination window

Age \& season adjusted SCCS excluding pre-vaccination window

SCRI with prior control interval

SCRI with posterior control interval

Unadjusted SCCS excluding all pre-vaccination time

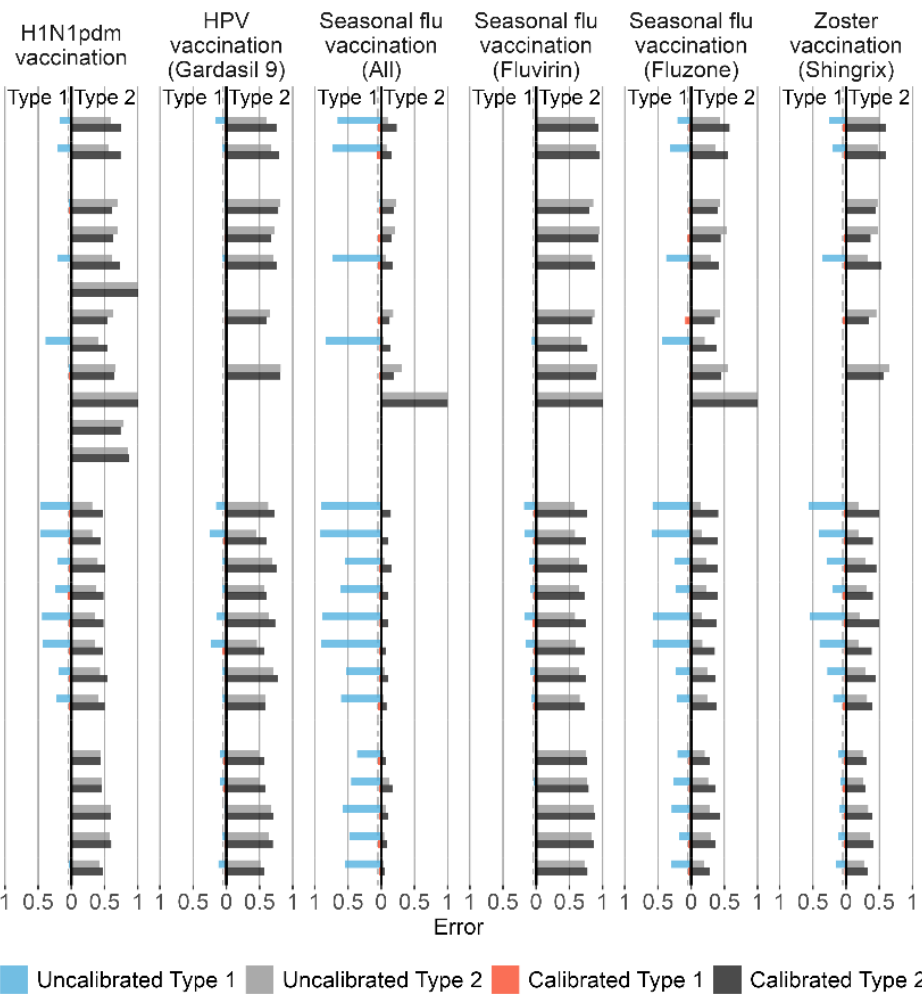

Fig. 4. Type 1 and 2 error before and after empirical calibration. For each method variation and vaccine group, the type 1 and 2 error before and after empirical calibration in the Optum EHR database are shown. The $x$-axis indicates the type 1 error (higher values to the left) and type 2 error (higher values to the right), based on the (calibrated) one-sided $p$-value. The dashed line indicates nominal type 1 error of 5\%. HPV = Human papillomavirus, PS = Propensity Score, SCCS = Self-Controlled Case Series, SCRI = SelfControlled Risk Interval, TaR = Time-at-Risk.

\subsection{Timeliness}

To evaluate timeliness, we divided our study periods into one-month intervals. For each month we executed the method variations on the data up to and including that month. We used MaxSPRT to account for the multiple testing of the same hypotheses each the month.[11] To facilitate comparison between methods we applied empirical calibration to restore type 1 error to nominal, and evaluate how many months must pass before type 2 error drops below $50 \%$, in other words until $50 \%$ of the positive controls exceed our alpha threshold of 0.05. Fig. 5 depicts the results of our timeliness analyses. Overall, the SCCS analyses were the most timely methods in most scenarios. Historical comparison methods were most timely in some cases, e.g. seasonal flu vaccination, and for larger true effect sizes. 


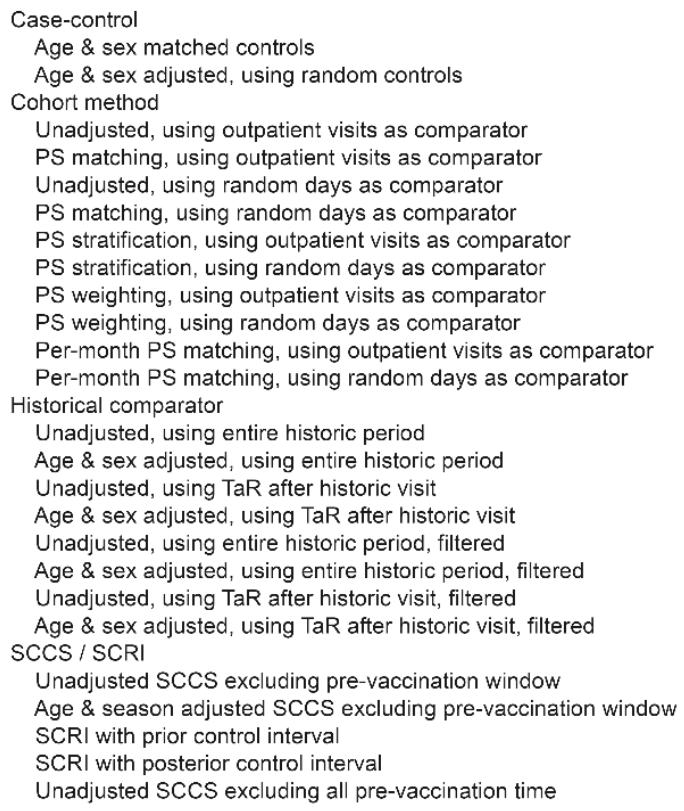

Unadjusted SCCS excluding pre-vaccination window Age \& season adjusted SCCS excluding pre-vaccination window SCRI with prior control interval

SCRI with posterior control interval

Unadjusted SCCS excluding all pre-vaccination time

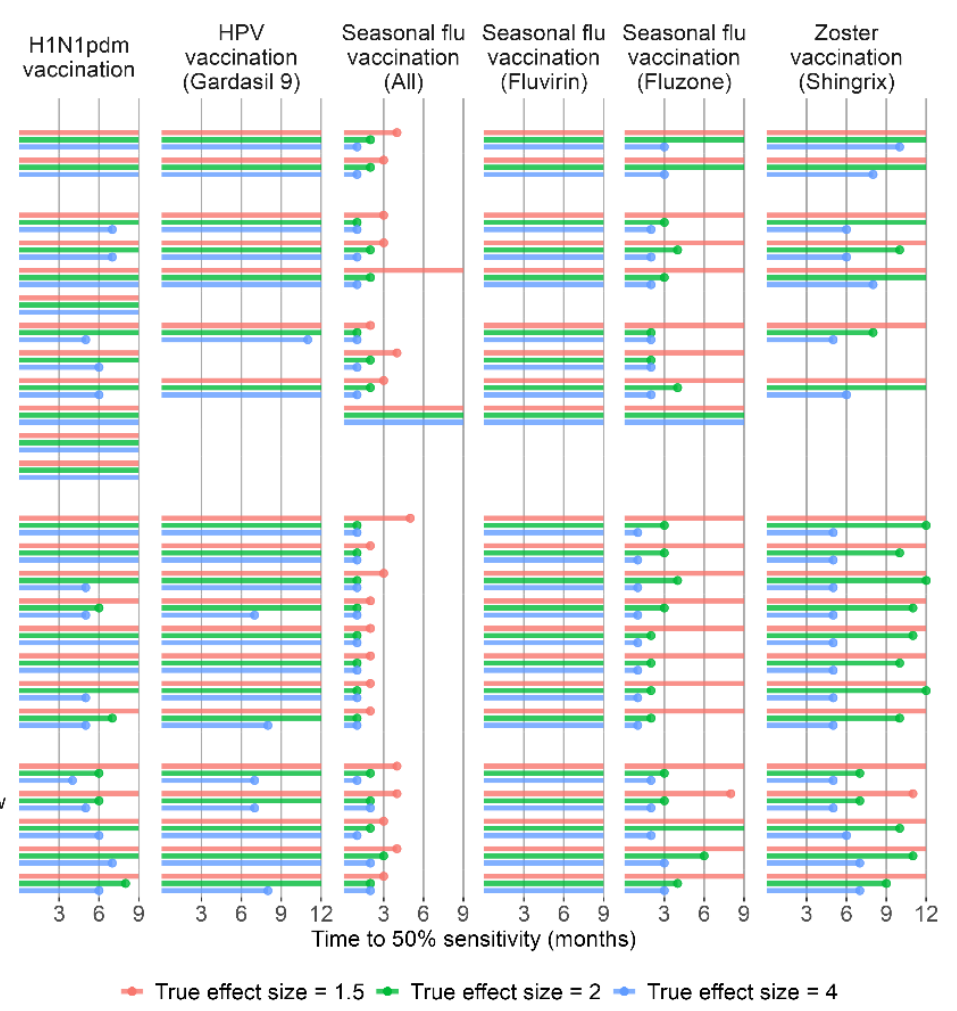

Fig. 5. Time to $50 \%$ sensitivity after calibration. For each method variation and vaccine group, the number of months of data needed to achieve $50 \%$ sensitivity based on the calibrated MaxSPRT in the Optum EHR database are shown, stratified by true effect size of the positive controls. HPV = Human papillomavirus, PS = Propensity Score, SCCS = Self-Controlled Case Series, SCRI = Self-Controlled Risk Interval, $\mathrm{TaR}=$ Time-at-Risk.

\section{Discussion}

\subsection{Key results}

Most methods show large type 1 error, often rejecting the null when the null is true. This is likely due to the systematic error inherent to observational research; probably not all confounding has been adequately adjusted for, and there may be measurement error in both exposures and outcomes as well as selection biases. When using negative controls to quantify this systematic error, we found it to be substantial in the case-control, historical comparator, and unadjusted cohort method designs, but much less in the other method variations. We hypothesize that the main reason for the systematic error may be due to uncontrolled confounding, caused by important differences in the comparator group versus the vaccinated group and therefore the underlying risk of the outcomes. Many of the positively biased analyses were unadjusted or only adjusted for age and sex. It appears these analyses missed important differences in which the vaccinated group is more vulnerable to disease outcomes of any kind, including outcomes potentially not caused by the vaccine (i.e. negative control outcomes). This would explain why self-controlled designs, such as the SCCS and SCRI, are less biased, because these are less vulnerable to between-person confounding.

The cohort method appears either positively or negatively biased, depending on the choice of comparator index date. If the index date is required to have an outpatient visit, the bias is negative, 
medRxiv preprint doi: https://doi.org/10.1101/2021.08.09.21261780; this version posted August 9, 2021. The copyright holder for this preprint (which was not certified by peer review) is the author/funder, who has granted medRxiv a license to display the preprint in perpetuity.

It is made available under a CC-BY 4.0 International license.

probably because the comparator group at index date is sicker or at least actively participating in the health care system and being captured in the database. In contrast, when using a random day as comparator index date the bias tends to be positive, possibly because the comparator group is healthier or may not seek health care in a way captured in the data. Also interesting is the fact that the cohort method appears to remain biased even when using PS that includes not only age and sex but all other variables available in the data, suggesting important confounders are completely missing from the data.

The varying levels of type 1 error hinder the ability to compare methods. A method showing low type 2 error, flagging most positive controls as signals, may be of limited interest if it also has high type 1 error, flagging most negative controls as signals. One way to facilitate comparison is by applying empirical calibration. Empirical calibration uses the systematic error distribution inferred from the negative control estimates to restore type 1 error to its nominal value. Depending on the amount of observed systematic error, this generally leads to increased type 2 error. After applying empirical calibration, results are mixed. For large effect sizes (e.g., incidence rate ratio $=4$ ), the historical comparator method is quickest to detect all positive controls, where this method's efficient design appears to compensate for and overcome its inherent systematic error. Smaller effect sizes can only be detected using methods that were already fairly unbiased to begin with, such as the SCCS design. Even in large databases such as Optum EHR and CCAE, no method was clearly capable of detecting positive controls with very small outcomes at an alpha of 0.05 . To allow detection of signals with such weak causal association in these databases will therefore require accepting a larger type 1 error. Larger databases or combinations of databases may lead to better detection performance, achieving shorter time to detection at lower type 1 error rates. Similarly, data with more complete information on important confounders, or data of a population where less confounding exists to begin with would improve performance, although it is unclear what type of data would meet these criteria. The four databases evaluated here showed comparable systematic error (see Supplementary Materials).

The results of our evaluation using real-world data agree to some extent to those in prior simulation studies, where SCCS was also observed to be both efficient and mostly unbiased.[7, 8] Although these prior studies indicated their unadjusted SCCS analysis was vulnerable to bias due to seasonal effects, in our study, SCCS analyses that did and did not adjust for seasonality both showed similar good performance. In contrast to the prior studies, we found the cohort method to be biased, possibly due to real data containing more unmeasured confounding than was simulated in those prior studies.

\subsection{Strengths and limitations of this study}

One of the main strengths of this study is the use of real-world data in addition to simulation of limited aspects (of positive controls), allowing the evaluation of methods in realistic scenarios. By using several different healthcare databases, we further improve the generalizability of our results.

Another strength is the inclusion of many different analysis variants, many of which have been used or proposed to be used for real vaccine safety surveillance studies. This includes more advanced designs, including SCCS adjusting for age and season using splines, and comparative cohort analyses using largescale propensity scores.

Whereas our negative control outcomes reflect real confounding, both measured and unmeasured, as well as measurement error, our positive controls simply assume that the same systematic error also applies when the true effect size is greater than 1 . In reality some forms of bias may change as a function of the true effect size, and this is not reflected in our imputed positive controls and therefore our type 2 error estimates. For example, if the TaR used in a method does not match the time when the 
medRxiv preprint doi: https://doi.org/10.1101/2021.08.09.21261780; this version posted August 9, 2021. The copyright holder for this preprint (which was not certified by peer review) is the author/funder, who has granted medRxiv a license to display the preprint in perpetuity.

It is made available under a CC-BY 4.0 International license .

risk of the outcome is increased by the vaccine, this can lead to bias towards the null, which is not reflected in our positive controls.

One final limitation of our study is that it included only vaccines for certain diseases in the past, and our results may not generalize to future vaccines for different diseases. For example, there are key features that are different between the situation surrounding the vaccines used as examples in this study and COVID-19 vaccines, such as the effects of nation-wide lockdowns, constraints in supply leading to highly targeted vaccinations, and decrease in regular patient care. For many vaccines, but especially for COVID19 vaccines, vaccinations may take place outside of regular healthcare and may therefore not be completely captured in the data.

\section{Conclusions}

When applying any method for vaccine safety surveillance we recommend considering the potential for systematic error, especially due to confounding, which for many designs appears to be substantial. Adjusting for age and sex alone is likely not sufficient to address the differences between vaccinated and unvaccinated, and the choice of index date plays an important role in the comparability of the groups. Inclusion of negative control outcomes allows both quantification of the systematic error and, if so desired, subsequent empirical calibration to restore type 1 error to its nominal value. To detect weaker signals, one may have to accept a higher type 1 error, either by not calibrating (in which case type 1 error will be higher than nominal but unknown), or by calibrating and raising the alpha threshold

What levels of type 1 and 2 error are acceptable will depend on many factors. A large number of false positives may erode societal confidence in a vaccine's safety without due cause, and exceed the downstream capacity of scientific and regulatory bodies to distinguish true positives from false positives. But false negatives, missing important safety signals, could have significant human cost.

\section{Acknowledgements}

We thank Paula Casajust and Carlos Areia for helping with the literature review and reviewing the manuscript for language.

\section{Funding}

UK National Institute of Health Research (NIHR), European Medicines Agency, Innovative Medicines Initiative 2 (806968), US Food and Drug Administration CBER BEST Initiative (75F40120D00039), and US National Library of Medicine (R01 LM006910).

\section{Declaration of Competing Interests}

All authors have completed the ICMJE uniform disclosure form at www.icmje.org/coi_disclosure.pdf and declare: GH receives grant funding from the US National Institutes of Health and the US Food \& Drug Administration. PBR, SF, and MJS are employees of Janssen Research and Development and shareholders in Johnson \& Johnson. DPA reports grants and other from Amgen, grants, non-financial support and other from UCB Biopharma, grants from Les Laboratoires Servier, outside the submitted work; and Janssen, on behalf of IMI-funded EHDEN and EMIF consortiums, and Synapse Management Partners have supported training programs organized by DPA's department and open for external participants. MAS receives grant funding from the US National Institutes of Health and the US Food \& Drug Administration and contracts from the US Department of Veterans Affairs and Janssen Research and Development. FN was an employee of AstraZeneca until 2019 and owns some AstraZeneca shares. NP receives grant funding from the Australian National Health and Medical Research Council

(GNT1157506). 
medRxiv preprint doi: https://doi.org/10.1101/2021.08.09.21261780; this version posted August 9, 2021. The copyright holder for this preprint (which was not certified by peer review) is the author/funder, who has granted medRxiv a license to display the preprint in perpetuity.

It is made available under a CC-BY 4.0 International license .

\section{Ethical approval}

The use of Optum and IBM Marketscan databases was reviewed by the New England Institution Review Board (IRB) and was determined to be exempt from broad IRB approval, as this research project did not involve human subjects research.

\section{Data sharing}

This study is part of the Evaluating Use of Methods for Adverse Event Under Surveillance (EUMAEUS) project. The protocol of this project is available at https://ohdsi-

studies.github.io/Eumaeus/Protocol.html, and we publicly host the source code at (https://github.com/ohdsi-studies/Eumaeus), allowing public contribution and review, and free re-use for anyone's future research.

\section{Supplementary materials}

One document containing

- List of abbreviations

- Vaccine exposure definitions

- Negative control outcomes

- Data source descriptions

- Description of evaluated methods

- Fitted systematic error distributions

- Type 1 and 2 error before and after calibration

- Time to $50 \%$ sensitivity after calibration

- Time to $80 \%$ sensitivity after calibration

- Type 1 and 2 error before and after calibration, stratified by true effect size

- Type 1 and 2 error before and after calibration and when adjusting for sequential testing, stratified by true effect size

- Area Under the Receiver-Operator-Curve (AUC)

- Performance metrics based on the effect-size estimates and confidence intervals.

- Effect-size estimates for negative control outcomes.

\section{References}

[1] Rosenthal S, Chen R. The reporting sensitivities of two passive surveillance systems for vaccine adverse events. American journal of public health. 1995;85:1706-9.

[2] Black S, Eskola J, Siegrist CA, Halsey N, MacDonald N, Law B, et al. Importance of background rates of disease in assessment of vaccine safety during mass immunisation with pandemic $\mathrm{H} 1 \mathrm{~N} 1$ influenza vaccines. Lancet (London, England). 2009;374:2115-22.

[3] Mesfin YM, Cheng A, Lawrie J, Buttery J. Use of routinely collected electronic healthcare data for postlicensure vaccine safety signal detection: a systematic review. BMJ global health. 2019;4:e001065.

[4] Tian Y, Schuemie MJ, Suchard MA. Evaluating large-scale propensity score performance through realworld and synthetic data experiments. International journal of epidemiology. 2018;47:2005-14. [5] Hripcsak G, Duke JD, Shah NH, Reich CG, Huser V, Schuemie MJ, et al. Observational Health Data Sciences and Informatics (OHDSI): Opportunities for Observational Researchers. Studies in health technology and informatics. 2015;216:574-8. 
medRxiv preprint doi: https://doi.org/10.1101/2021.08.09.21261780; this version posted August 9, 2021. The copyright holder for this preprint (which was not certified by peer review) is the author/funder, who has granted medRxiv a license to display the preprint in perpetuity.

It is made available under a CC-BY 4.0 International license .

[6] Leite A, Andrews NJ, Thomas SL. Near real-time vaccine safety surveillance using electronic health records-a systematic review of the application of statistical methods. Pharmacoepidemiology and drug safety. 2016;25:225-37.

[7] McClure DL, Glanz JM, Xu S, Hambidge SJ, Mullooly JP, Baggs J. Comparison of epidemiologic methods for active surveillance of vaccine safety. Vaccine. 2008;26:3341-5.

[8] Glanz JM, McClure DL, Xu S, Hambidge SJ, Lee M, Kolczak MS, et al. Four different study designs to evaluate vaccine safety were equally validated with contrasting limitations. Journal of clinical epidemiology. 2006;59:808-18.

[9] Schuemie MJ, Ryan PB, Hripcsak G, Madigan D, Suchard MA. Improving reproducibility by using highthroughput observational studies with empirical calibration. Philosophical transactions Series A, Mathematical, physical, and engineering sciences. 2018;376.

[10] Tian Y, Schuemie MJ, Suchard MA. Evaluating large-scale propensity score performance through real-world and synthetic data experiments. International journal of epidemiology. 2018.

[11] Kulldorff M, Davis RL, Kolczak+ M, Lewis E, Lieu T, Platt R. A Maximized Sequential Probability Ratio Test for Drug and Vaccine Safety Surveillance. Sequential Analysis. 2011;30:58-78.

[12] Silva IR, Kulldorff M. The 'Sequential' R package. 3.3.1 ed2021.

[13] Schuemie MJ, Hripcsak G, Ryan PB, Madigan D, Suchard MA. Empirical confidence interval calibration for population-level effect estimation studies in observational healthcare data. Proceedings of the National Academy of Sciences of the United States of America. 2018;115:2571-7.

[14] Schuemie MJ, Ryan PB, DuMouchel W, Suchard MA, Madigan D. Interpreting observational studies: why empirical calibration is needed to correct p-values. Statistics in medicine. 2014;33:209-18. 